\title{
LA CALIDAD DE LA EDUCACIÓN PÚBLICA: ¿UN PROBLEMA DE QUIÉN?
}

\author{
Ana Maria Gutiérrez Huby \\ Docente-Facultad de Ciencias Contables
}

\begin{abstract}
RESUMEN
La universidad pública nuevamente pasa por una de sus tantas crisis educativas y económicas, las cuales le impiden avanzar hacia la consecución de objetivos académicos de alta calidad. .

Pero, a pesar del reconocimiento de la crisis y de, en algunos casos, su falta de calidad, diferentes sectores de la sociedad hacen esfuerzos muy grandes por la creación de un sistema que se encargue de la evaluación, acreditación y la certificación de la calidad educativa, ya que el Perú no cuenta aún con un sistema para la acreditación de su educación superior.

Es toás, aunque la Ley General de Educación garantiza su funcionamiento, no existen señales políticas claras que permaitan inferir que el Ejecutivo o el Legislativo tengan la voluntad de crear los organismos que velen por la calidad de la educación superior peruana. No existen en el Perú mecanismos que permitan comparar la homogeneidad de la formación de profesionales de la misma especialidad en dos universidades públicas distintas.
\end{abstract}

El Perú, como uno de los tantos países latinoamericanos que permanentemente están sumergidos en la ingobernabilidad, muestra cifras alarmantes de desempleo y subempleo, producto de su falta de planeación educativa. Por ejemplo, si analizamos la evolución del crecimiento universitario observamos que en 1980 existían en el Perú 35 universidades y este número se incrementó a 56 en 1994 y a 78 en el 2002.

En 1980, de las 35 universidades que existían, 25 eran públicas; en 1994 de las 56 universidades, 28 eran públicas; y en el 2002, de las 78 universidades 46 eran privadas. Pero a pesar de este importante incremento de universidades privadas, las públicas siguen albergando el mayor número de alumnos $(59 \%$ de 1 población estudiantil).

Según estudios realizados por el CONCYTEC, la investigación y el desarrollo (I\&D) en nuestro país representa el 0,11\% del PBI, una de las más bajas de la región y bastante rezagadas respecto de países como Brasil, Cuba, Chile y Argentita, que tienen un coeficiente de 1,05\%;0,62\%;0,56\% y 0,42\%, respectivamente.

En el Perú, en los últimos 25 años, la inversión en I\&D ha descendido de 0,36\% del PBI en 1975 a 0,10\% del PBI en el 2002. La inversión pública en I\&D es alrededor del 55\% del total de la inversión y el resto es originada en el sector privado; sin embargo, si se analiza por sectores institucionales se puede observar que las universidades en su conjunto representan aproximadamente el $42 \%$ del total de la inversión, siendo muy similar los tiveles en las universidades públicas y privadas.

Según la Asamblea Nacional de Rectores, en el año 2000, el gasto promedio por cada estudiante de universidad pública en el Perú fue equivalente a 780 dólares, mientras que el promedio para el resto de países en América Latina fue de 937 dólares y de 5595 dólares en Estados Unidos, según datos de la UNESCO para 1998. 
Entre los años 1991 y 2002, el número de postulantes a universidades públicas aumentó en $66 \%$, asimismo en el 2002 postularon 229107 jóvenes a universidades públicas, mientras que 62149 lo hicieron a universidades privadas.

Palabras clave: Educación superior, calidad educativa, universidad pública, inversión pública educativa.

\section{INTRODUCCIÓN}

$¿$ A cuántas personas llega la universidad hoy?, ¿cuántos queremos que accedan?, ¿en qué tiempo?, ¿cuál es el horizonte para su expansión? Son preguntas que hacen referencia a una política de educación; su formulación debe equipararse con la agenda del desarrollo y su integración al sistema educativo nacional.

No se puede pensar en un desarrollo integral educativo sin tener una planificación del sector; $y$ por ese motivo, hace falta desarrollar un proyecto global educativo. Asimismo, la reforma se tiene que encarar con sentido social, no solo como resultado de algo comercial.

Cómo podemos crear una universidad pública moderna con los problemas económicos por los que atraviesa la educación. Tenemos autoridades que tienen discurso positivo hacia la educación, pero no los ponen en práctica.

Tenemosqueobservarpuescon unavisión objetiva la crisis universitaria, porque todos los que estamos en el sistema universitario público nos encontramos afectados, querámoslo o no, por los grandes cambios científicos y tecnológicos. No se puede pensar en cambios puntualesen pocotiempo. Setiene que lograr un consenso mínimo para lograr un sistema educativo de calidad.

Si se piensa que estudiar en una universidad pública es una salida razonablemente económica para las crisis permanentes que viven nuestro país, podríamos decirque estamos probablemente escogiendo una justificable salida a una problemática que presentan muchas familias. Los títulos universitarios no garantizan un buen empleo, pero mejoran notablementelas posibilidades de conseguirlo.

\section{DEMANDA EDUCATIVA}

La demanda por la educación superior a partir de la década del 70 a la actualidad se haincrementado significativamente, a pesar de las prolongadas recesiones, el empobrecimiento masivo de la población, la altísima desocupación, la precarización del trabajo y el descenso de los ingresos de los trabajadores (particularmente de los no calificados).

Los universitarios graduados muestran porcentajes de desempleo menores de aquellos sectores donde no se ha alcanzado este nivel de formación. Si seguimos esta líneade pensamiento, diríamos entonces que hay una estrategia política pública para enfrentar la explosión de la demanda de estudios superiores en nuestro país. Esta estrategia ha consistido en la disminución de trabas para la creación de nuevas universidades, tanto públicas como privadas, pero, con la consiguiente disminución de calidad en todas sus áreas y procesos.

Cada universidad nacional es receptora de una subvención global del Estado que surge de la repetición automática de 10 
históricamente percibido y de lo obtenido vía negociaciones particulares. No se cuenta con un consenso entrelos actores del sistema universitario sobre un criterio objetivo de distribución (como podría ser el número de alumnos, costos diferenciales por carrera, tasa de graduación u otros). Observaremos entonces que en un futuro al presentarse mayores restricciones fiscales, sería previsible un aumento de los conflictos distributivos entre las instituciones del sistema educativo nacional.

En este sentido, es importante remarcar que los criterios para la asignación presupuestariacobran mayorimportanciaen un contexto de escasez. Al respecto, cabe señalar que la subordinación del nivel de calidad a la asignación de partidas presupuestarias a las universidades públicas puedegenerar, entonces, un efecto perverso, es decir contribuir a cristalizar y, aún más, a ampliar las desigualdades de la calidad de las instituciones.

Las mejores universidades obtendrían más beneficios, mientras que se congelaría el financiamiento a las de menor calidad, terminando por apoyar su desaparición en vez de su mejoramiento. No obstante, una Dolíticadefinanciamiento porcalidad puede apoyar la introducción de compensaciones $\checkmark$ poner su peso en el enfoque del mejoramiento institucional.

Por esta razón, toda política pública debe partir de un buen diagnóstico que señale no solamente los problemas a resolver sino las causas de los mismos y sus manifestaciones nás significativas.

\section{CALIDAD EDUCATIVA}

Es evidente que la baja calidad de la educación pública en el caso de nuestro país está relacionada, además de los insuficientes recursos financieros, con los recursos humanos, físicos, tecnológicos e infor máticos que carecen de calidad en la aplicación educativa que es de amplia cobertura.

Adicionalmente, si observamos, veremos que existen elementos socioculturales que afectan negativamente la calidad de nuestra educación, tales como la ausencia de una sólida investigación y el aislamiento de nuestro sistema de educación pública a las corrientes internacionales que le dan vigencia y actualidad a la educación universitaria.

En la educación superior pública, la inteligencia nacional juega un papel importante. Por lo tanto, el país tiene la obligación de velar porque la educación se entregue con lacalidad, eficaciay pertinencia que son deseables. La importancia de la educación superior públicase refleja, además de su nivel de investigación, en el volumen de recursos humanos y materiales que las instituciones manejan, los que son insuficientes en relación con lo idealmente deseable; por ello, es importante que se haga un uso óptimo y transparente de ellos.

Silasinstituciones de educación superior pública, de acuerdo a su perfil, realizaran adecuadamente sus funciones y prepararan personas altamente calificadas para los diversos sectores de la actividad académica, económica, social y política, si generaran conocimientos y desarrollaran tecnología, si preservaran y difundieran cultura; entonces, la inversión social que se hace en ellas estaría plenamente justificada.

Sin embargo, tenemos que aceptar que la búsqueda de la calidad tiene muchas facetas y va más allá de una estrecha 
interpretación del rol académico de las diferentes instituciones o programas; por lo tanto, implica, además, prestar atención a la formación con calidad de estudiantes, a la optimización de infraestructura y a la visualización con calidad del entorno académico.

De la misma manera en que se generan estas exigencias hacia las entidades universitarias públicas, la sociedad debe asegurar entonces un acceso racional y planificado a la educación superior a través de una oferta adecuada con calidad y magnitud que se ajusten a las necesidades de desarrollo económico y social que el país requiere.

\section{EL ACCESO A LA UNIVERSIDAD PÚBLICA CON CALIDAD}

$\mathrm{El}$ acceso racional y planificado a una universidad pública implicaría la búsqueda de calidad, eficacia y equidad en la distribución de las oportunidades investigativas y profesionalizantes, impacto en su ámbito social y eficacia en la utilización de los recursos disponibles (humanos y financieros) que, por definición, son escasos en un país de menor desarrollo como el nuestro.

Si todos estos aspectos relacionados con lacalidad y, unidos a un interés por una buena administración ybuengobierno representaran un papel importante en la manera cómo funcionan determinadas instituciones de educación superior pública, la forma en que serían evaluadas, la imagen institucional que proyectaran hacia la comunidad académica y la sociedad en general harían pensar que se trataría de instituciones académica y administrativamente organizadas, que responden de manera eficaz y consistente a los exigentes retos que el futuro le plantea en los campos de acción que les son propios. mas no es así.

\section{ANÁlisis DEL MODELO DE GESTIÓN DE LA UNIVERSIDAD PÚBLICA}

Analizando el modelo de gestión en la universidad pública, se observa que el liderazgo a su interior es vital en su conducción hacia el logro de objetivos y para cristalizar la realización de su misión. Por ello, es necesario dotar a estas instituciones de un modelo que debe contemplar, por lo menos los siguientes aspectos:

- Fortalecimiento de los procesos de planeación que tengan en cuenta el presente, pero que incluyan una visión prospectiva y de futuro.

- Modernizar los sistemas de infor mación con la incorporación de la infor mática y los sistemas de costos.

- Realizar acciones permanentes de autoevaluación de la gestión que per mitan no solo examinar los productos y resultados, sino los procesos y los procedimientos para apreciar el funcionamiento de la institución como un sistema vivo $y$, deter minar tareas estratégicas y programas de mejoramiento continuo.

- Incorporar el concepto de profesionalización de la gestión institucional, donde los funcionarios del aparato administrativo de las instituciones de educación superior pública, en sus diferentes instancias académico-administrativas, deben poseer una calificación específica que los capacite para el ejercicio de su labor.

- Elaborar indicadores de gestión como instrumentos que permitan no solo el 
direccionamiento de los esfuerzos de la gestión, sino, particularmente, la autoevaluación permanente delagestión.

\section{CONCLUSIÓN}

En conclusión, la cultura de la autoevaluación y del mejoramientocontinuo deberá encontrar, paulatinamente, un espacio en la comunidad universitaria pública que permita que la organización institucional sea sometida a un proceso de renovación y modernización de su estructura y funcionamiento.

Estos requisitos son necesarios para que sea más efectiva y pueda asumir con mayor fir meza su compromiso con la calidad, para ser más flexible y per meable a los cambios y a la aplicación de las técnicas modernas de gestión que le permitan incorporar procesos de planeación, evaluación y control del desempeño, y resultados.

Asimismo, es requisito importante que tenga presente la necesidad de involucrarlos conceptos de autoevaluación, acreditación, mejoramiento continuo y productividad en todos sus procesos, para conseguir que los programas y proyectos se constituyan en herramientasquearticulen la planeación con la debida inversión, que facilite la asignación eficaz de los recursos presupuestales, consolidando su sistema de control interno y racionalizando los procedimientos, y simplificando los trámites que le permitan cumplir con éxito el rol que la sociedad les ha asignado.

\section{REFERENCIAS}

1. Becerra, O. "Notas para construir el debate sobre financiamiento de las Universidades". En: Revista Escenarios Alternatizas N. ${ }^{\circ}$ 12. Año 5, 2001.

2. Coraggio J. "Construir universidad en la adversidad”. Mimeo, 2001.

3. Del Bello J.C. "Desafíos de la política de la educación superior en América Latina”. Reflexiones a partir del caso argentino con énfasis en la evaluación para el mejoramiento de la calidad". Mimeo, 2001.

4. Kisilevsky, M. "Condiciones sociales y pedagógicas de ingreso a la educación superior". IIPE, mimeo, 2002.

5. EduCalidad. Iniciativas para la calidad de la educación superior No 1, mayo 2004 - Perú. GICES.

6. EduCalidad. Iniciativas para la calidad de la educación superior N. 3, 2004 -Perú. GICES.

7. EduCalidad. Iniciativas para la calidad de la educación superior N. ${ }^{\circ}$ 5, 2004 - Perú. GICES. 